\title{
Grand canonical approach to modeling hydrogen trapping at vacancies in $\alpha$-Fe
}

\author{
E. R. M. Davidson, ${ }^{1}$ T. Daff, ${ }^{2}$ G. Csanyi, ${ }^{2}$ and M. W. Finnis $\circledast^{1}$ \\ ${ }^{1}$ Department of Materials and Department of Physics, Thomas Young Centre, Imperial College London, Exhibition Road, \\ SW7 2AZ London, United Kingdom \\ ${ }^{2}$ Engineering Laboratory, University of Cambridge, Cambridge CB2 1PZ, United Kingdom
}

(Received 15 November 2018; accepted 14 May 2020; published 22 June 2020)

\begin{abstract}
Vacancies in iron are hydrogen traps, important in the understanding of hydrogen embrittlement of steel. We present a grand canonical approach to computing the trap occupancy as a function of both temperature and hydrogen concentration from practically zero to supersaturation. Our method couples a purpose-made machine-learned H-Fe potential, which enables rapid sampling with near-density-functional-theory accuracy, with a statistical mechanical calculation of the trap occupancy using the technique of nested sampling. In contrast to the conventional assumption (based on Oriani theory) that at industrially relevant hydrogen concentrations and ambient conditions vacancy traps are are fully occupied, we find that vacancy traps are less than fully occupied under these conditions, necessitating a reevaluation of how we think about "mobile hydrogen" in iron and steel.
\end{abstract}

DOI: 10.1103/PhysRevMaterials.4.063804

\section{INTRODUCTION}

The propensity of hydrogen to embrittle steels is an important practical problem, motivating physicists, electrochemists, and corrosion engineers to understand the absorption, trapping, and diffusion of hydrogen, its devastating effect on ductility, and how it can be controlled. Although a number of mechanisms for embrittlement have been put forward, their relative importance is still a matter of ongoing research and debate [1-5]. Whatever the particular mechanism of embrittlement, hydrogen traps, including precipitates, dislocations, and vacancies, may play a protective role by sequestering the hydrogen [6]. The possibility of this has recently been observed by atomic probe microscopy of deuterium in carbide particles [7]. Static calculations have strongly suggested that a single vacancy in $\alpha$-Fe can trap up to six $\mathrm{H}$ atoms [8-10], although the marginal trapping energy of the sixth is very small. The maximum number was challenged by Sugimoto and Fukai [11], who concluded from their Monte Carlo simulations that it should be five, a conclusion supported by an earlier DensityFunctional Theory (DFT) calculation by Tateyama and Ohno [12]. The lowering of vacancy-formation energy by hydrogen trapping is the origin of the superabundant vacancy effect discovered by Fukai et al. [13]. Trapping by vacancies and the superabundant vacancy effect have been investigated by many authors since, for a wide range of metals [14-22]. This formation energy lowering was described by Kirchheim $[15,16]$ as a "defactant" effect, by analogy with Gibbs' original theory of surfactants. The defactant effect is a step in at least one of the proposed embrittlement mechanisms, sometimes referred to as hydrogen-enhanced strain-induced vacancy formation $[1,23]$. We know that the solubility of hydrogen in iron is very low [24] but that under operating conditions it only requires less than $1 \mathrm{ppm}$ dissolved in the crystalline matrix of a steel to cause embrittlement. In the present paper, we are addressing the question of how many hydrogens would be sequestered in a vacancy under realistic conditions. The point here is not whether the maximum number at low temperature is five or six but how the mean number trapped varies with temperature and the bulk concentration or, equivalently, chemical potential.

A simple "lattice model" was proposed by Oriani [25], which represents the bcc Fe by discrete tetrahedral interstitial sites and the vacancy by six trap sites, corresponding to the static equilibrium locations of the $\mathrm{H}$, which in the vacancy are close to sites that in the perfect crystal are octahedral. The average occupancy of a vacancy can then be expressed as

$$
\left\langle n_{v}\right\rangle=\frac{6 y e^{-\beta \Delta E}}{1+y e^{-\beta \Delta E}},
$$

where $y$ is the fractional lattice concentration, $\beta=1 /\left(k_{B} T\right)$, and $\Delta E$ is the binding energy of the vacancy trap relative to a lattice site. More sophisticated lattice models, which include the distinct configurations of multiple hydrogens within a vacancy and the interactions between them have been developed, a good example for our specific case of bcc Fe is the work of Tateyama and Ohno [12]. In the case of $\mathrm{Ni}$, the vibrational contribution to the free energy of the atomic nuclei has been included within a quasiharmonic approximation [21] but the major limitation of a lattice model remains, namely, that the $\mathrm{H}$ atoms are confined to discrete sites. To the best of our knowledge, the only model that also included the full contribution to the free energy from off-lattice movement of the hydrogen is that of Tanguy and Mareschal [14], and Tanguy et al. [26], for $\mathrm{Al}$ and $\mathrm{Ni}$, respectively. For $\mathrm{Al}$, they employed an off-lattice Monte-Carlo (OLMC) approach, with an Embedded-Atom Model (EAM) of the Al-H interaction to evaluate the superabundant vacancy effect. For $\mathrm{Ni}$, they also made detailed calculations with DFT for $\mathrm{H}$ clusters in vacancies, where $\mathrm{H}$ was located on the various high-symmetry sites, but used OLMC and and an EAM potential to estimate the off-lattice contribution.

For the case of interest here, the weak binding energy $(<0.1 \mathrm{eV})[27,28]$ to the lowest energy, tetrahedral sites in $\mathrm{Fe}$ 
suggests that in reality $\mathrm{H}$ moves smoothly and swiftly in a continuous landscape of potential even at room temperature, implying that its total entropy in the bulk will be significantly greater than the configurational entropy captured by the lattice model. For the same reason, its entropy when trapped in a vacancy will also be greater, so the net effect of the lattice approximation on trap occupancy is unclear. How to calculate the hydrogen occupancy of a trap using a simple yet accurate approach beyond the lattice model is the challenge that we address here.

Density-functional theory $[29,30]$ is the most popular firstprinciples method for computing the energies and energy barriers, due to its good compromise between computational cost and accuracy. However, for sampling configurational phase space including a vacancy, it is too computationally demanding. Empirical interatomic potentials are much faster but their accuracy and transferability are always questionable. A possible solution to this problem is to create a moderately large data set of DFT calculations on configurations strictly relevant to the problem at hand, and then fit a potential with a very flexible functional form, which can reliably predict the energetics of the $\mathrm{H}-\mathrm{Fe}$ system including a vacancy, but without necessarily being transferable to very different configurations, such as surfaces. Recently, various neural networks, Gaussian process regression, and similar approaches have been applied to create such potentials [31-34]. Here we introduce a Gaussian approximation potential (GAP) for the $\mathrm{H}-\alpha$-Fe interaction.

The statistical mechanical solution to the problem of trap occupancy can be formulated in terms of a grand canonical ensemble and a partition function, the calculation of which requires sampling the potential energy surface, weighted by a Boltzmann factor. For our scenarios in which the bulk concentration is very low, we can conveniently express the result in terms of readily evaluated canonical partition functions, as we show below.

A number of methods have become widely used to enable the efficient sampling of potential energy surfaces, including metadynamics [35], Wang-Landau sampling [36], parallel tempering [37,38], and nested sampling [39,40]. We have chosen the latter approach because it allows the direct calculation of the partition function itself, which is essential to allow access to the regime of very low $\mathrm{H}$ concentrations. The problem with low $\mathrm{H}$ concentrations arises due to the supercell approximation (a small periodically repeating block of unit cell of crystal containing a single vacancy). The very low probability of finding a $\mathrm{H}$ atom anywhere within a supercell which allows accurate DFT-quality calculations is very low, leading to very poor sampling of the relative probability of the $\mathrm{H}$ being trapped or not. But we can turn the low $\mathrm{H}$ concentration to our advantage by sampling separately the configuration space of a single $\mathrm{H}$ atom within a relatively small supercell of perfect crystal, which enables us to calculate the chemical potential of an arbitrarily dilute, ideal solution of $\mathrm{H}$ in Fe. This chemical potential is then used as the formal boundary condition for the grand canonical sampling of $\mathrm{H}$ trapped by the vacancy, which is thus insensitive to the definition of the supercell that contains the vacancy. Since our approach considers both the full phase space of the bulk and vacancy regions of the system and includes H-H interactions, we obtain far more

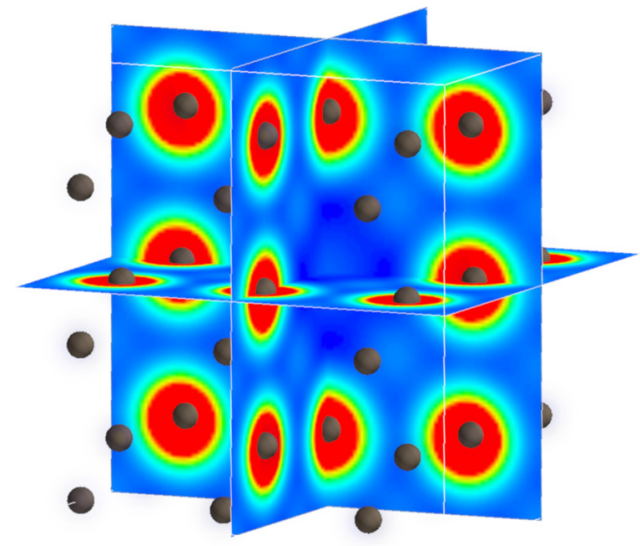

FIG. 1. Representation of 53 -atom $(3 \times 3 \times 3)$ supercell with a vacancy at the center. The potential energy for a single $\mathrm{H}$ atom in the cell is represented by the heat maps on the cross sections. The six trap sites correspond to the dark-blue regions.

realistic results than the lattice model, and can comment on the effects of the approximations made in the Oriani approach. This combination of a machine-learned potential with nested sampling and the grand canonical ensemble should be useful for analyzing other trapping problems.

\section{MODELS FOR CALCULATING ENERGIES AND FORCES}

We model the system using a 54-atom cubic supercell $(3 \times 3 \times 3$ body-centered unit cells), with periodic boundary conditions. It is well known that in bulk Fe, the fourfold coordinated tetrahedral sites are the preferred sites for interstitial $\mathrm{H}$ atoms $[27,28]$ and we have confirmed this, noting, however, that they are very shallow traps, separated by a barrier of only about $88 \mathrm{meV}$. Figure 1 shows the potential energy surface of $\mathrm{H}$ in the presence of a Fe vacancy, with six equivalent trap positions, close to the location of the cube face-centers of the body-centered unit cell (octahedral sites).

In all the calculations in this paper, the positions of the $\mathrm{Fe}$ atoms are fixed after an initial DFT relaxation of the vacancy and so there is no need to model the Fe subsystem, only its interaction with $\mathrm{H}$. We now give a brief overview of the GAP methodology that we use. A more extensive discussion is found in papers reporting recent applications [32,41-46] and a review [47]. The key idea is to posit interatomic functional forms that are highly flexible due to a large number of parameters (universal, in the limit) but avoid overfitting because the least-squares problem that we solve to obtain the parameters is highly regularized, resulting in smooth potential functions. This still leaves a lot of freedom for designing potentials, in particular in the choice of representation of the atomic geometry. We find that the customary decomposition of the total energy into two-body, three-body, etc. terms is very helpful. The two-body functions are parametrized by the interatomic distance, whereas the three-body terms are described by a symmetrized combination of the three distances. For the basis functions, we choose Gaussians, because they achieve our dual goals of being universal approximators and are intuitive to parametrize. This formalism was successfully 
used recently for the low body-order terms of potentials for amorphous carbon [46] and boron [45]. Whereas, in those cases, a many-body term was also needed, we found that in the present case, just two- and three-body terms are sufficient. We found it sufficient to include explicit three-body terms for the interaction of an $\mathrm{H}$ atom with two Fe neighbors, and there are no explicit three-body terms for the interaction of two $\mathrm{H}$ atoms and a $\mathrm{Fe}$ atom or three $\mathrm{H}$ atoms.

The interaction between pairs of $\mathrm{H}$ atoms, which reduces the magnitude of the trap energy as the vacancy fills, is described by a Yukawa potential with a screening length of $1.5 \AA^{-1}$. The interaction energy between a $\mathrm{H}$ atom and a collection of $\mathrm{Fe}$ atoms is given by

$$
E_{\mathrm{H}-\mathrm{Fe}}\left(r_{\mathrm{H}}\right)=\sum_{i \in\{\mathrm{Fe}\}} V_{2}\left(\left|r_{\mathrm{H}}-r_{i}\right|\right)+\sum_{j<k \in\{\mathrm{Fe}\}} V_{3}\left(r_{\mathrm{H}}, r_{j}, r_{k}\right)
$$

Within the GAP framework, the two-body potential $V_{2}$ is written as a linear combination of kernel basis functions,

$$
V_{2}(r)=\sum_{m} x_{2, m} K_{2}\left(r, r_{m}\right)
$$

where the coefficients $x_{2, m}$ are to be fitted and we choose a Gaussian kernel $K_{2}\left(r, r_{m}\right)=\exp \left(-\left|r-r_{m}\right|^{2} / 2 \theta_{2}^{2}\right)$ with width parameter $\theta_{2}=1.5 \AA$. The potential is smoothly taken to zero at $r=6 \AA$.

The three-body term, $V_{3}$, describes the interaction of a $\mathrm{H}$ atom $(i)$ with two Fe neighbors ( $j$ and $k$ ), and is constructed similarly to the two-body potential, except that its arguments are explicitly made symmetric with respect to swapping $\mathrm{Fe}$ atoms. The new symmetrized coordinates are

$$
\begin{gathered}
q_{1}=r_{i j}+r_{i k}, \\
q_{2}=\left(r_{i j}-r_{i k}\right)^{2}, \\
q_{3}=r_{j k},
\end{gathered}
$$

collected into a vector $\mathbf{q}=\left(q_{1}, q_{2}, q_{3}\right)$, and so the symmetrized three-body term $\tilde{V}_{3}$ is given by

$$
V_{3}\left(r_{i}, r_{j}, r_{k}\right) \equiv \tilde{V}(\mathbf{q})=\sum_{m} x_{3, m} K_{3}\left(\mathbf{q}, \mathbf{q}_{m}\right),
$$

where again the coefficients $x_{3, m}$ are to be fitted, $K_{3}$ is a three-dimensional Gaussian with three independent length scale parameters, which were set to be $1 / 2$ of the extent of the training data in each dimension. The cutoff in this case was $5 \AA$. The training data for the $\mathrm{H}-\mathrm{Fe}$ interaction potential comprises snapshots from molecular dynamics trajectories of 54 and $128 \mathrm{Fe}$ atoms with either 0,1 , or $2 \mathrm{Fe}$ atoms removed, and a single $\mathrm{H}$ atom added. Plane-wave DFT calculations were performed for each configuration with and without the $\mathrm{H}$ atom, and the interaction energy defined as the difference between the two total energies. All parameter settings of the DFT calculations were identical to those reported for pure Fe calculations by Dragoni et al. [48]. Altogether, about 400 configurations were used in the fit, comprising about $28 \mathrm{k}$ atoms. The fit is obtained as the analytic solution to a linear leastsquares problem, where the target data is the H-Fe interaction energy and corresponding gradient. The GAP framework uses Tikhonov regularization; we set the magnitude to $10^{-5} \mathrm{eV}$ and $10^{-4} \mathrm{eV} / \AA$ for the energy and forces, respectively. The mean absolute errors of the fit on an independent test set were $20 \mathrm{meV}$ for energies and $10 \mathrm{meV} / \AA$ for force components. For comparison, the mean absolute errors of the EAM model of Ref. [49] for the H-Fe interaction on the same set of configurations are $250 \mathrm{meV}$ for energies and $730 \mathrm{meV} / \AA$ for force components. The resulting potentials for the two and three-body interaction energies are shown in Fig. 2. We used the QUIP software package [50] to obtain the fit and its PYTHON API to subsequently calculate the potential and its gradients. More details and numerical values of the parameters for these potentials can be downloaded [51]. Employing the interatomic potential enables us to compute the energy of a configuration at nearly DFT accuracy in around $100 \mathrm{~ms}$ on a single CPU core using our naive implementation of the two- and threebody functions. The same calculation would take around 15 minutes on 24 cores of the current United Kingdom national supercomputer. This already represents an enormous savings in terms of both time and cost and was critical in enabling us to perform nested sampling calculations. The above potential functions could easily be tabulated and used within a software package such as LAMMPS, thereby obtaining significant further increases in speed.

\section{SAMPLING}

Our chosen method for the statistical mechanics sampling is nested sampling, originally developed by Skilling to estimate the Bayesian evidence for model comparison purposes $[39,40]$. The approach was adapted by Pártay et al. to compute directly and efficiently the partition function in atomistic problems, and it enables us to compute any thermodynamic property as a function of temperature from a single run
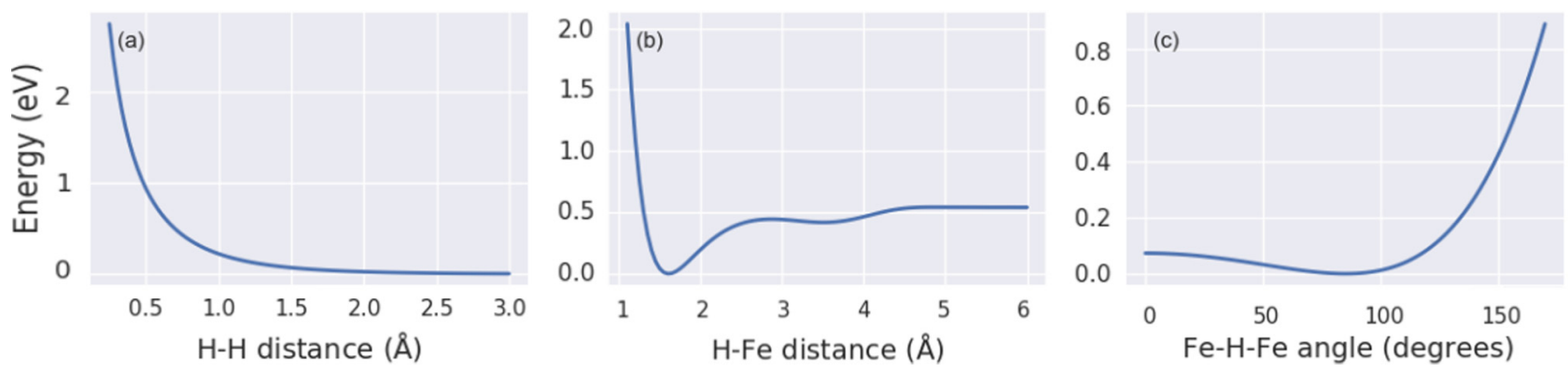

FIG. 2. The two- and three-body interaction energies used in this paper. The Yukawa potential for the H-H interaction energy is shown in (a); (b) shows the two-body GAP H-Fe potential, and (c) shows the three-body GAP Fe-H-Fe potential upon varying the angle. 
[52]. Briefly, a set of configurations $x_{n}$ of decreasing energy $E_{n}=E\left(x_{n}\right)$ is generated with the property that the ratio of phase space volumes enclosed by successive energy level sets $E_{n}$ is approximately constant, $\alpha=K /(K+1)$, where $K$ is the number of samples, held constant. We start by choosing $K$ configurations randomly, and in iteration $n$, the highest energy sample is assigned as $x_{n}$, and is replaced by a new sample that is randomly chosen from among the others, cloned, and then decorrelated by a bounded random walk in which we reject any move that would result in an energy greater than $E_{n}$. At the end, the canonical partition function is estimated (up to a multiplicative constant) as

$$
Q_{C}(T) \simeq \sum\left(\alpha^{n}-\alpha^{n+1}\right) e^{-\beta E\left(x_{n}\right)} .
$$

To test convergence with respect to the number of walkers, $K$, we ran four independent copies of each simulation. We found that 192 walkers were sufficient.

All our nested sampling calculations were carried out with the PYMATNEST package [53].

\section{EVALUATING THE GRAND CANONICAL PARTITION FUNCTION}

Despite the efficiency gains introduced by the methods above, direct calculation of thermodynamic properties in the canonical ensemble at low concentrations (<100 ppm) of $\mathrm{H}$ that are damaging in real systems would be impractical due to the large size of the simulation cell required ( $>10^{4}$ atoms). We can nevertheless compute the expected number of $\mathrm{H}$ atoms in the vacancy at any concentration by conceptually separating the vacancy region from the bulk region and treating the former with a variable number of $\mathrm{H}$ atoms within a grand canonical ensemble. The phrase "in the vacancy" implies that we can define a boundary to the vacant lattice site. The position of this boundary is in principle arbitrary, but for practical purposes, as long as the bulk concentration is sufficiently low, the number of $\mathrm{H}$ atoms deemed to be inside the vacancy will not be significantly changed by the choice of this boundary within reasonable limits. This can easily be verified in the case considered in this paper, for which the borders of a $3 \times 3 \times 3$ supercell are a suitable boundary. It contains 54 atomic sites. Suppose the bulk concentration of $\mathrm{H}$ were $10^{-4}$ per atom, which is the largest value we consider. Assuming a Poisson distribution, it is easy to show that less than $0.5 \%$ of such bulk supercells would be occupied by any hydrogen and less than $0.3 \%$ of those that are would be occupied by more than one atom of hydrogen. This leads us to consider the $\mathrm{H}$ content of a bulk supercell compared to one of the same size containing a vacancy, with which the hydrogen is in equilibrium. If the bulk concentration is only significantly perturbed at the atoms neighboring the vacant site, then the mean hydrogen content of the supercell $\left\langle n_{v}\right\rangle$ would be equal to the excess hydrogen in the vacancy to within 0.005 atoms, which is more than adequate for our purposes. Longer ranged strain-fields will introduce a small error in our estimates, which would be of minor importance in practice. Thus it is unnecessary, besides physically meaningless, to define a bounding surface any closer to the vacant site than the borders of the supercell. This is further justified in the Appendix.
To derive the required formula for computation, we adopt from the start a notation which defines discrete states in energy or space, labeled with subscript $i$, summations over which can be equated to integrals weighted by a density of states. The expectation value for the number of $\mathrm{H}$ atoms $n_{v}$ in the vacancy in the grand canonical ensemble can be written

$$
\left\langle n_{v}\right\rangle=\frac{1}{Q_{G C}} \sum_{n_{v}} \sum_{i} n_{v} g_{i} e^{-\beta E_{i, n_{v}}+\beta n_{v} \mu},
$$

where the grand canonical partition function is defined by

$$
Q_{\mathrm{GC}}=\sum_{n_{v}} \sum_{i} g_{i} e^{-\beta E_{i, n_{v}}+\beta n_{v} \mu} .
$$

$E_{i, n_{v}}$ and $g_{i}$ are the energies and degeneracies of states we denote as inside the vacancy, respectively, $\mu(T, y)$ is the chemical potential of hydrogen to be calculated in the bulk reservoir, in which $y$ denotes the bulk concentration in units to be discussed below. We emphasize that inside the vacancy means inside a rather small supercell containing the vacancy, the exact size of which is arbitrary. $\beta$ is $1 /\left(k_{\mathrm{B}} T\right)$, where $k_{\mathrm{B}}$ and $T$ are the Boltzmann constant and temperature. Equation (9) can be evaluated over the regimes of interest by writing it in terms of the canonical partition function,

$$
Q_{C}^{v a c}\left(T, n_{v}\right)=\sum_{i} g_{i} e^{-\beta E_{i, n_{v}}},
$$

which we can substitute into Eq. (9) to give

$$
\left\langle n_{v}\right\rangle=\frac{1}{Q_{\mathrm{GC}}} \sum_{n_{v}} n_{v} Q_{C}^{\mathrm{vac}}\left(T, n_{v}\right) e^{\beta n_{v} \mu},
$$

in which

$$
Q_{\mathrm{GC}}=\sum_{n_{v}} Q_{C}^{\mathrm{vac}}\left(T, n_{v}\right) e^{\beta n_{v} \mu} .
$$

This manipulation conveniently splits our task into two distinct parts. First, we need to calculate $\mu(T, y)$ for any low concentration $y$ of bulk $\mathrm{H}$ and, second, we need calculations of $Q_{C}^{\mathrm{vac}}\left(T, n_{v}\right)$ for integer values of $n_{v}$ from zero to six (more than six will not be relevant). We can then combine the two kinds of calculation into formula Eq. (12). The calculation of $Q_{C}^{\mathrm{vac}}$ is the task of our nested sampling, so it remains to derive an expression for $\mu$.

Assuming zero pressure, we shall evaluate the Helmholtz free energy of a dilute concentration $y$ hydrogen atoms per Fe atom. Let us divide a perfect bulk crystal into $N$ identical space-filling cells, each containing $N_{a}$ atoms of Fe. Normally, we have in mind here the supercells with periodic boundary conditions, as used in DFT calculations, in which $1 \leqslant N_{a} \leqslant$ 54. We can apply the following approach both to a lattice model, in which only tetrahedral sites are occupied, or the continuous phase space which is used in this paper. The thermodynamic limit of $N \rightarrow \infty$ will be taken.

We suppose the bulk hydrogen to be distributed over these cells such that there is at most one $\mathrm{H}$ atom per cell, which is an excellent approximation for our purposes as indicated above. The number of Fe atoms is $N_{a} N$ and the number of $\mathrm{H}$ atoms is $n=y N_{a} N$, which is equal to the number of supercells occupied by a hydrogen atom. The total partition function $Z_{C}$ for the perfectly crystalline bulk system (remember we are neglecting here the movement of the Fe lattice) can therefore 
be factorized into the partition function of $n$ singly occupied cells and the partition function for the choice of these $n$ cells from the total $N$ :

$$
Z_{C}=Q_{C}^{\text {bulk }}\left(T, 1 / N_{a}\right)^{n} \frac{N !}{(N-n) ! n !} .
$$

The chemical potential will be obtained from this expression via the standard thermodynamic formula:

$$
\mu=-k_{\mathrm{B}} T \frac{\partial}{\partial n} \ln Z_{C}
$$

We make the usual Stirling's approximation for the factorials in Eq. (13), namely,

where

$$
\ln \left(\frac{N !}{(N-n) ! n !}\right) \rightarrow N S(n / N),
$$

$$
S(x)=-x \ln (x)-(1-x) \ln (1-x),
$$

which is accurate in our thermodynamic limit. Inserting this into Eq. (14), the derivative is straightforward and we obtain

$$
\mu=k_{\mathrm{B}} T \ln \frac{n / N}{1-n / N}-k_{\mathrm{B}} T \ln Q_{C}^{\text {bulk }}\left(T, 1 / N_{a}\right) .
$$

Replacing $n / N$ by $y N_{a}$, this can be written in terms of the concentration per host atom in the form

$$
\mu=k_{\mathrm{B}} T \ln \left(y N_{a}\right)-k_{\mathrm{B}} T \ln Q_{C}^{\text {bulk }}\left(T, 1 / N_{a}\right),
$$

where we have assumed again $y N_{a} \ll 1$ ito make the replacement $1-N_{a} y \rightarrow 1$ in the first term. Consider now the calculation of the second term using a supercell and periodic boundary conditions with nested sampling or any other sampling technique. As long as $N_{a}$ is big enough to avoid spurious interactions between the single $\mathrm{H}$ atom and its periodic images, it is clear that

$$
Q_{C}^{\text {bulk }}\left(T, 1 / N_{a}\right)=N_{a} Q_{C}^{\text {bulk }}(T, 1) .
$$

This linear scaling ensures that $N_{a}$ cancels out of expression Eq. (18), which we can finally write as

$$
\mu=k_{\mathrm{B}} T \ln (y)-k_{\mathrm{B}} T \ln Q_{C}^{\mathrm{bulk}}(T, 1),
$$

from which

$$
\exp (\beta \mu)=y / Q_{C}^{\text {bulk }}(T, 1) .
$$

The grand canonical partition function for hydrogen in the vacancy can now be expressed as

$$
Q_{\mathrm{GC}}=\sum_{n_{v}} Q_{C}^{\mathrm{vac}}\left(T, n_{v}\right)\left(y / Q_{C}^{\mathrm{bulk}}(T, 1)\right)^{n_{v}}
$$

and the mean number of trapped atoms is

$$
\left\langle n_{v}\right\rangle=\frac{1}{Q_{\mathrm{GC}}} \sum_{n_{v}} n_{v} Q_{C}^{\mathrm{vac}}\left(T, n_{v}\right)\left(y / Q_{C}^{\mathrm{bulk}}(T, 1)\right)^{n_{v}} .
$$

Both canonical partition functions are computed by nested sampling, which we apply to the full energy landscape and, for comparison purposes only, to the lattice model, both with and without $\mathrm{H}-\mathrm{H}$ interactions. Taking the reference state for hydrogen to be when it is at rest in a bulk tetrahedral site, Eq. (12) still applies to the discrete lattice models, with the simplification that $Q_{C}^{\text {bulk }}(T, 1)$ reduces to six. For the six vacancy trapping sites, in our lattice model calculations we take the nine nondegenerate energies for the states of $1 \leqslant$ $n_{v} \leqslant 6$ from our static DFT calculations. The effect of the $\mathrm{H}-\mathrm{H}$ interactions is to split the degeneracy of all the states with $2 \leqslant n_{v} \leqslant 5$. While the single vacancy with a few $\mathrm{H}$ atoms comprise a relatively simple system in terms of composition, size, and geometry, the same approach could be applied to any system in which a solute has a low concentration in the bulk, so the defective and perfectly crystalline regions can be considered separately using the nested sampling method and linked via the explicit calculation of the partition functions and the chemical potential.

\section{RESULTS}

The average number of $\mathrm{H}$ atoms in a vacancy is shown in Fig. 3(a) as a function of temperature for a range of hydrogen concentrations, calculated using nested sampling, the grand canonical approach, and the GAP model for the potential energy surface. For comparison, we show the same for the lattice model in Fig. 3(b). The expected occupancy of the vacancy using Oriani's theory, which does not include $\mathrm{H}-\mathrm{H}$ interactions, is shown in Fig. 3(c). From these results, we can estimate the temperature at which the vacancy goes from being fully to half occupied (six $\mathrm{H}$ to three $\mathrm{H}$ ). Our model [Fig. 3(a)] indicates this happens at $340 \mathrm{~K}$ for a bulk $\mathrm{H}$ concentration of $0.01 \mathrm{ppm}$, or $500 \mathrm{~K}$ for the very high concentration of $10 \mathrm{ppm}$. The reduced phase space of the lattice model [Fig. 3(b)] spuriously enhances the trapping, so the corresponding temperatures are 380 and $630 \mathrm{~K}$. By ignoring $\mathrm{H}-\mathrm{H}$ repulsion, we further increases the apparent occupancy of the vacancy, bringing the temperature of halfoccupancy up to 450 and $750 \mathrm{~K}$ for $0.01 \mathrm{ppm}$ and $10 \mathrm{ppm}$, respectively [Fig. 3(c)]. As noted before, we kept the positions of Fe atoms fixed in the DFT optimal configuration. Although certainly possible within the same methodological framework, allowing movement of $\mathrm{Fe}$ atoms is not expected to change our conclusions significantly, since their vibrations are nearly harmonic at room temperature and their contribution to configurational entropy is much smaller than that of the mobile $\mathrm{H}$ atoms.

\section{CONCLUSIONS}

Using a purpose-made hydrogen-iron interaction potential defined by Gaussian process regression fitted to DFT calculations, we applied nested sampling to obtain the mean number of $\mathrm{H}$ atoms trapped in a vacancy in an $\alpha$-Fe crystal, as a function of bulk hydrogen concentrations from $10^{-4}$ down to $10^{-11}$. To deal with such low bulk hydrogen concentrations, we introduce a grand-canonical formulation, which requires a separate calculation of the chemical potential of $\mathrm{H}$ as a function of its bulk concentration. We obtained this chemical potential from nested sampling calculations on a single $\mathrm{H}$ atom in the perfect crystal. Our grand canonical approach to dilute solutions does not suffer from the difficulty of simulation methods for which the required supercell volume scales inversely with the concentration of solute. 


\section{(a)}

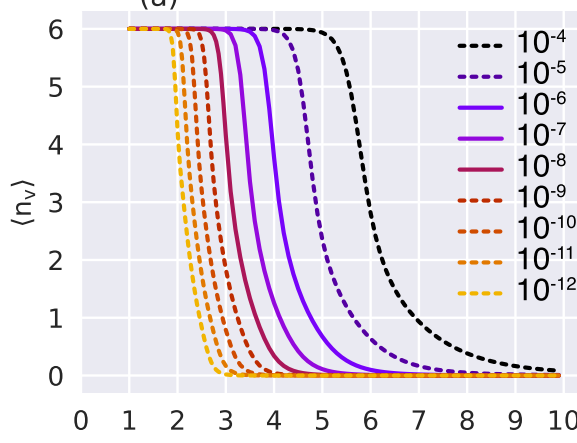

(b)

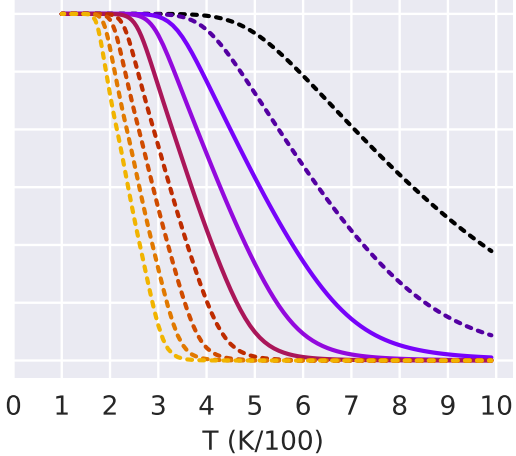

(c)

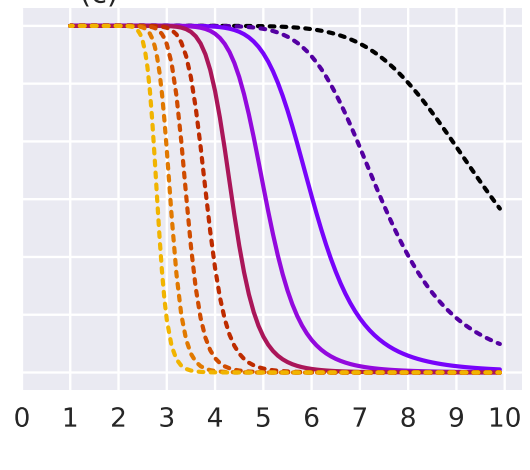

FIG. 3. Expected number of $\mathrm{H}$ atoms in the vacancy as a function of temperature for a range of bulk $\mathrm{H}$ concentrations, calculated using (a) the main model presented in this paper, (b) the same nested sampling approach applied to the discrete lattice system, in which the energies are only required for $\mathrm{H}$ atoms on the bulk tetrahedral sites and the six sites in the vacancy, and (c) the Oriani model, using the same single $\mathrm{H}$ on-site energies as (b), but switching off the $\mathrm{H}-\mathrm{H}$ interactions. The concentrations are in units of atom fraction. The three solid lines represent concentrations most relevant to industrially observed $\mathrm{H}$ embrittlement of steels.

At sufficiently low temperature, the saturated vacancy trap for all bulk concentrations contains six H. Our calculations indicate that this maximum level of trapping would not be achieved at around room temperature for bulk concentrations below 0.1 atom ppm. It is widely accepted that the propensity for steels to embrittle is correlated with the amount of "mobile hydrogen" rather than the total including those in deep traps such as carbides, grain boundaries, etc. and thus understanding the nature and dynamics of mobile hydrogen is in turn critical for understanding hydrogen embrittlement. The designation of "mobile hydrogen" is conventionally taken to be equivalent to $\mathrm{H}$ atoms in bulk interstitial lattice sites, and sometimes referred to as lattice hydrogen. Our results, however, suggest that the $\mathrm{H}$ atoms trapped at vacancies contribute to the pool of mobile hydrogen in the system. Thus, theories of hydrogen dynamics in iron and steel, including analyses of thermal desorption spectroscopy (the main analytical tool in the field) such as Kissinger theory [54], likely have to be revised. There will be further direct implications to theories of $\mathrm{H}$ embrittlement that involve the interaction with vacancies explicitly, such as hydrogen-enhanced strain-induced vacancy formation [1].

For the present paper, we assumed classical nuclei, which would be a poor approximation for hydrogen in metals much below room temperature. The approach could readily be extended to include nuclear quantum effects for $\mathrm{H}$ using the Feynman path-integral formalism which replaces the $\mathrm{H}$ atoms with ring-polymer chains. See, for example, Gillan et al. [55] for a very clear exposition. We similarly ignored movement of $\mathrm{Fe}$ atoms, and diffusion leading to clustering of the $\mathrm{Fe}$ monovacancies. The latter would lead to a variety of other trapping configurations, from divacancies upward, which we have not discussed here. The same treatment could be applied to all these aspects of the problem, using the GAP model for Fe-Fe of Dragoni et al. [48]. Our combination of machine-learned interatomic potentials and nested sampling, together with our grand canonical approach to trap occupancy, should be useful for many similar questions involving segregation and trapping of impurities at interfaces or dislocations.

\section{ACKNOWLEDGMENTS}

We acknowledge the support of the UK EPSRC under the programme Grant HEmS, No. EP/ L014742, and an anonymous referee who helped us to greatly improve an earlier version of this paper.

\section{APPENDIX: TEST OF THE 1 H/SUPERCELL APPROXIMATION}

To appreciate better our approximation of no more than one $\mathrm{H}$ atom per perfect supercell, we can demonstrate it with the lattice model, assuming an ideal solution of $\mathrm{H}$ on a lattice of tetrahedral sites, of which there are six per Fe atom. In particular, we examine the error made by our approximation as a fraction of the exact entropy in the ideal solution.

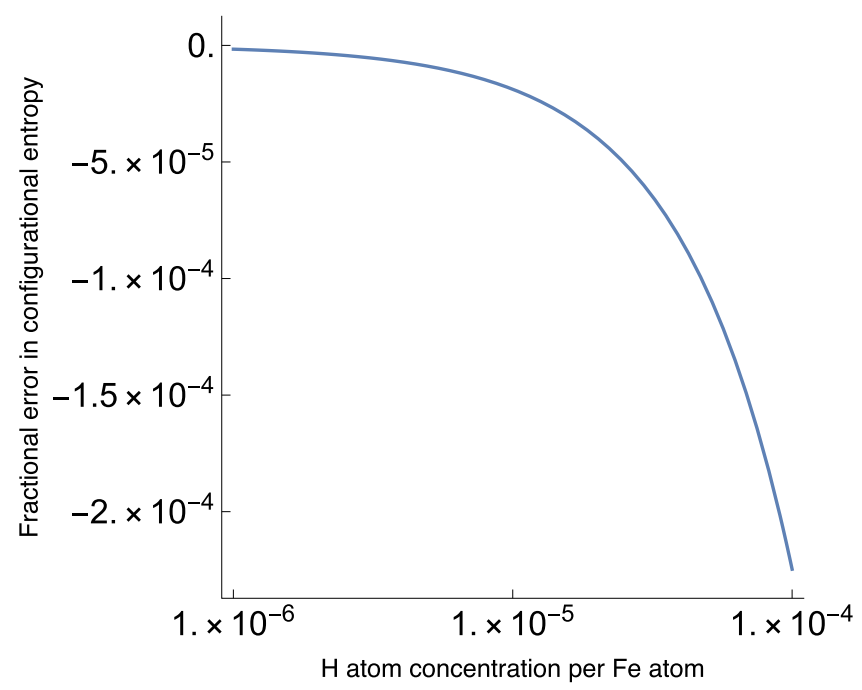

FIG. 4. Estimate of the fractional error in the approximated configurational entropy of bulk hydrogen with our one-H-per-supercell model. This error estimate is based on the lattice approximation, in which $\mathrm{H}$ can only occupy tetrahedral sites. 
We make use of the function $S(x)$, defined in Eq. (15), which is the familiar dilute solution expression for the entropy per site when the fractional site occupancy is $x$. We also work with the number of atoms per supercell, $N_{a}$, and the number of sites per atom, $N_{s}$, which in this example are to be 54 and six, respectively, and the number of $\mathrm{H}$ atoms per host atom is $y \ll 1$, as above. The "exact" dilute solution entropy per atom is then

$$
S_{\text {exact }}=N_{s} S\left(y / N_{s}\right) .
$$

In the lattice model, our approximate partition function Eq. (13) becomes

$$
Z_{C}^{\text {lattice }}=\left(N_{s} N_{a}\right)^{n} \frac{N !}{(N-n) ! n !}
$$

From which the entropy per atom is

$$
\begin{aligned}
S_{\text {approx }} & =\frac{1}{N N_{a}} \ln Z_{C}^{\text {lattice }} \\
& =\frac{n}{N N_{a}} \ln \left(N_{s} N_{a}\right)+\frac{1}{N_{a}} S(n / N) \\
& =y \ln \left(N_{s} N_{a}\right)+\frac{1}{N_{a}} S\left(y N_{a}\right) .
\end{aligned}
$$

We have made use of the identity $y N_{a}=n / N$ to eliminate the extensive quantities $N$ and $n$. The error is evaluated as $S_{\text {approx }}-S_{\text {exact }}$. It is easy to see by Taylor expanding in $y$ that the terms to leading order as $y \rightarrow 0$, namely, $y \ln y$, cancel, and the fractional error is very small until $y$ exceeds $10^{-4}$. This is shown graphically in Fig. 4.
[1] M. Nagumo, Hydrogen related failure of steels-a new aspect, Mater. Sci. Technol. 20, 940 (2004)

[2] Y. Fukai, The Metal-Hydrogen System: Basic Bulk Properties, Vol. 21 (Springer-Verlag, Berlin Heidelberg, 2005).

[3] T. Neeraj, R. Srinivasan, and J. Li, Hydrogen embrittlement of ferritic steels: Observations on deformation microstructure, nanoscale dimples and failure by nanovoiding, Acta Mater. 60, 5160 (2012).

[4] R. Gangloff and B. Somerday (Eds.), Gaseous Hydrogen Embrittlement of Materials in Energy Technologies: The Problem, its Characterisation and Effects on Particular Alloy Classes (Woodhead Publishing Limited, Cambridge, 2012).

[5] A. T. Paxton, A. P. Sutton, and M. W. Finnis (Eds.), The Challenges of Hydrogen and Metals, Philosophical Transactions A: Mathematical, Physical and Engineering Sciences Vol. 375 (The Royal Society, London, 2017).

[6] J. Takahashi, K. Kawakami, and T. Tarui, Direct observation of hydrogen-trapping sites in vanadium carbide precipitation steel by atom probe tomography, Scr. Mater. 67, 213 (2012).

[7] Y.-S. Chen, D. Haley, S. S. Gerstl, A. J. London, F. Sweeney, R. A. Wepf, W. M. Rainforth, P. A. Bagot, and M. P. Moody, Direct observation of individual hydrogen atoms at trapping sites in a ferritic steel, Science 355, 1196 (2017).

[8] E. Hayward and C.-C. Fu, Interplay between hydrogen and vacancies in alpha-Fe, Phys. Rev. B 87, 174103 (2013).

[9] D. Mirzaev, A. Mirzoev, K. Y. Okishev, A. Shaburov, G. Ruzanova, and A. Ursaeva, Formation of hydrogen-vacancy complexes in alpha iron, Phys. Met. Metallogr. 113, 923 (2012).

[10] K. Ohsawa, K. Eguchi, H. Watanabe, M. Yamaguchi, and M. Yagi, Configuration and binding energy of multiple hydrogen atoms trapped in monovacancy in bcc transition metals, Phys. Rev. B 85, 094102 (2012).

[11] H. Sugimoto and Y. Fukai, Hydrogen-induced superabundant vacancy formation in bcc fe: Monte Carlo simulation, Acta Mater. 67, 418 (2014).

[12] Y. Tateyama and T. Ohno, Stability and clusterization of hydrogen-vacancy complexes in alpha-Fe: An ab initio study, Phys. Rev. B 67, 174105 (2003).
[13] Y. Fukai and N. Ōkuma, Evidence of copious vacancy formation in Ni and Pd under a high hydrogen pressure, Jpn. J. Appl. Phys. 32, L1256 (1993).

[14] D. Tanguy and M. Mareschal, Superabundant vacancies in a metal-hydrogen system: Monte carlo simulations, Phys. Rev. B 72, 174116 (2005).

[15] R. Kirchheim, Reducing grain boundary, dislocation line and vacancy formation energies by solute segregation. I. Theoretical background, Acta Mater. 55, 5129 (2007).

[16] R. Kirchheim, On the solute-defect interaction in the framework of a defactant concept, Int. J. Mater. Res. 100, 483 c (2009).

[17] L. Ismer, M. S. Park, A. Janotti, and C. G. Van de Walle, Interactions between hydrogen impurities and vacancies in $\mathrm{Mg}$ and Al: A comparative analysis based on density functional theory, Phys. Rev. B 80, 184110 (2009).

[18] R. Nazarov, T. Hickel, and J. Neugebauer, First-principles study of the thermodynamics of hydrogen-vacancy interaction in fcc iron, Phys. Rev. B 82, 224104 (2010).

[19] R. Nazarov, T. Hickel, and J. Neugebauer, Ab initio study of H-vacancy interactions in fcc metals: Implications for the formation of superabundant vacancies, Phys. Rev. B 89, 144108 (2014).

[20] L. Bukonte, T. Ahlgren, and K. Heinola, Thermodynamics of impurity-enhanced vacancy formation in metals, J. Appl. Phys. 121, 045102 (2017).

[21] A. Metsue, A. Oudriss, and X. Feaugas, Thermodynamic vs. kinetic origin of superabundant vacancy formation in Ni single crystals, Corrosion 75, 898 (2019).

[22] J. Hou, X.-S. Kong, X. Wu, J. Song, and C. Liu, Predictive model of hydrogen trapping and bubbling in nanovoids in bcc metals, Nat. Mater. 18, 833 (2019).

[23] R. Kirchheim, Revisiting hydrogen embrittlement models and hydrogen-induced homogeneous nucleation of dislocations, Scr. Mater. 62, 67 (2010).

[24] J. D. Silva and R. B. Mclellan, The solubility of hydrogen in super-pure-iron single crystals, J. Less-Common Met. 50, 1 (1976).

[25] R. A. Oriani, The diffusion and trapping of hydrogen in steel, Acta Metall. Mater. 18, 147 (1970). 
[26] D. Tanguy, Y. Wang, and D. Connetable, Stability of vacancyhydrogen clusters in nickel from first-principles calculations, Acta Mater. 78, 135 (2014).

[27] D. E. Jiang and E. A. Carter, Diffusion of interstitial hydrogen into and through bcc fe from first principles, Phys. Rev. B 70, 064102 (2004).

[28] D. C. Sorescu, First principles calculations of the adsorption and diffusion of hydrogen on fe (100) surface and in the bulk, Catal. Today 105, 44 (2005).

[29] P. Hohenberg and W. Kohn, Inhomogeneous electron gas, Phys. Rev 136, B864 (1964).

[30] W. Kohn and L. J. Sham, Self-consistent equations including exchange and correlation effects, Phys. Rev 140, A1133 (1965).

[31] J. Behler and M. Parrinello, Generalized neural-Network Representation of High-Dimensional Potential-Energy Surfaces, Phys. Rev. Lett. 98, 146401 (2007).

[32] A. P. Bartók, M. C. Payne, R. Kondor, and G. Csányi, Gaussian Approximation Potentials: The Accuracy of Quantum Mechanics, without the Electrons, Phys. Rev. Lett. 104, 136403 (2010).

[33] A. P. Thompson, L. P. Swiler, C. R. Trott, S. M. Foiles, and G. J. Tucker, Spectral neighbor analysis method for automated generation of quantum-accurate interatomic potentials, J. Comput. Phys. 285, 316 (2015).

[34] A. V. Shapeev, Moment tensor potentials: A class of systematically improvable interatomic potentials, Multiscale Model. Simul. 14, 1153 (2016).

[35] C. Micheletti, A. Laio, and M. Parrinello, Reconstructing the Density of States by History-Dependent Metadynamics, Phys. Rev. Lett. 92, 170601 (2004).

[36] F. Wang and D. P. Landau, Efficient, Multiple-Range Random Walk Algorithm to Calculate the Density of States, Phys. Rev. Lett. 86, 2050 (2001).

[37] R. H. Swendsen and J.-S. Wang, Replica Monte Carlo Simulation of Spin-Glasses, Phys. Rev. Lett. 57, 2607 (1986).

[38] D. Frantz, D. L. Freeman, and J. Doll, Reducing quasiergodic behavior in Monte Carlo simulations by J-walking: Applications to atomic clusters, J. Chem. Phys. 93, 2769 (1990).

[39] J. Skilling, Nested sampling, AIP Conf. Proc. 735, 395 (2004).

[40] J. Skilling, Nested sampling for general Bayesian computation, Bayesian Anal. 1, 833 (2006).

[41] S. Fujikake, V. L. Deringer, T. H. Lee, M. Krynski, S. R. Elliott, and G. Csányi, Gaussian approximation potential modeling of lithium intercalation in carbon nanostructures, J. Chem. Phys. 148, 241714 (2018)
[42] S. T. John and G. Csanyi, Many-body coarse-grained interactions using Gaussian approximation potentials, J. Phys. Chem. B 121, 10934 (2017).

[43] A. P. Bartók and G. Csányi, Gaussian approximation potentials: A brief tutorial introduction, Int. J. Quantum Chem. 115, 1051 (2015).

[44] P. Rowe, G. Csányi, D. Alfè, and A. Michaelides, Development of a machine learning potential for graphene, Phys. Rev. B 97, 054303 (2018).

[45] V. L. Deringer, C. J. Pickard, and G. Csányi, Data-Driven Learning of Total and Local energies in Elemental Boron, Phys. Rev. Lett. 120, 156001 (2018).

[46] V. L. Deringer, C. Merlet, Y. Hu, T. H. Lee, J. A. Kattirtzi, O. Pecher, G. Csányi, S. R. Elliott, and C. P. Grey, Towards an atomistic understanding of disordered carbon electrode materials, Chem. Commun. 54, 5988 (2018).

[47] M. Ceriotti, M. J. Willatt, and G. Csányi, Machine Learning of Atomic-Scale Properties Based on Physical Principles, in Handbook of Materials Modeling: Methods: Theory and Modelling, edited by A. W. and Y. S. (Springer, Cham, 2018), pp. 1-27.

[48] D. Dragoni, T. D. Daff, G. Csányi, and N. Marzari, Achieving DFT accuracy with a machine-learning interatomic potential: Thermomechanics and defects in bcc ferromagnetic iron, Phys. Rev. Mater. 2, 013808 (2018)

[49] A. Ramasubramaniam, M. Itakura, and E. A. Carter, Interatomic potentials for hydrogen in $\alpha$-iron based on density functional theory, Phys. Rev. B 79, 174101 (2009).

[50] QUIP (2017, Last checked 08 May 2020), http://github.com/ libatoms/quip.

[51] See Supplemental Material at http://link.aps.org/supplemental/ 10.1103/PhysRevMaterials.4.063804 for more details and values of the parameters for these potentials.

[52] L. B. Pártay, A. P. Bartók, and G. Csányi, Efficient sampling of atomic configurational spaces, J. Phys. Chem. B 114, 10502 (2010).

[53] PYMATNEST, 2016 (accessed May 8, 2020), http://github. com/libatoms/pymatnest.

[54] H. E. Kissinger, Variation of peak temperature with heating rate in differential thermal analysis, J. Res. Natl. Bur. Stand. 57, 217 (1956).

[55] M. J. Gillan, The path-integral simulation of quantum systems, in Computer Modelling of Fluids Polymers and Solids, edited by C. R. A. Catlow, C. S. Parker, and M. P. Allen (Kluwer Academic Publishers, Dordrecht, 1990), Vol. 293, pp. 155-188. 